\title{
Effect of different inclusion levels of oil palm fronds on in vitro rumen fermentation pattern, fatty acid metabolism and apparent biohydrogenation of linoleic and linolenic acid.
}

\begin{abstract}
Effects of different inclusion levels of oil palm fronds (OPF) on rumen fermentation, apparent biohydrogenation of linoleic (C18:2n-6) and linolenic (C18:3n-3) acid and their biohydrogenation intermediates are described after $24 \mathrm{~h}$ in vitro incubations with buffer (20 $\mathrm{ml})$, rumen fluid $(5 \mathrm{ml})$ and standard dairy concentrate $(0.250,0.225,0.200$ and $0.175 \mathrm{~g})$. Four inclusion levels of $\operatorname{OPF}(0,0.025,0.050$ and $0.075 \mathrm{~g})$ were tested in two in vitro incubation series: without (Experiment 1) and with (Experiment 2) addition of a mixture of sunflower $(10 \mathrm{mg})$ and linseed oil $(10 \mathrm{mg})$ as an external polyunsaturated fatty acid source. Increasing inclusion levels of OPF changed the rumen fermentation pattern in both in vitro incubation series, in terms of a decreased production of short chain fatty acids, a linear increase in acetate and a decrease in propionate proportions. A trend for lower amounts of C18:2n-6 and C18:3n-3 were observed due to a higher apparent biohydrogenation rate of the latter, as the inclusion levels of OPF increased. In order to maximize the valorisation of OPF as a roughage source in Malaysian ruminant production systems, further technological research is needed to improve its digestibility.
\end{abstract}

Keyword: Biohydrogenation; Fatty acid metabolism; Oil palm fronds; Rumen fermentation. 